Check for updates

Cite this: RSC Adv., 2020, 10, 31243

\title{
Development of a portable lab-on-a-valve device for making primary diagnoses based on gold- nanoparticle aggregation induced by a switchable linker
}

\begin{abstract}
Jungwoo Hahn, (D) $\dagger^{b}$ Eunghee Kim, (ID $\dagger^{a}$ Hyebin Han (D) and Young Jin Choi (D) *abc
We have developed a low-cost, portable lab-on-a-valve (LOV) integrated microdevice for the detection of pathogens in primary-diagnosis settings. This system was designed for field-based pathogen detection based on the aggregation of gold nanoparticles induced by a switchable linker. A three-way valve, which has attracted much attention as a functional mesofluidic platform for pressure-driven flow, has been designed as a universal reaction platform that combines the functions of fluid flow and a reaction chamber. In addition, we obtain rapid and enhanced visual signals by the use of a syringe filter to remove gold nano-aggregates (Au NAs). Using this device, Salmonella Typhimurium down to $10^{1} \mathrm{CFU} \mathrm{mL}^{-1} \mathrm{can}$ be visually detected within $30 \mathrm{~min}$ by performing a simple operation that requires no complex equipment. This prototype device has great potential for use in the semi-quantitative and qualitative identification of pathogens in on-site primary diagnoses.
\end{abstract}

Received 10th June 2020

Accepted 15th August 2020

DOI: $10.1039 / \mathrm{d} 0 \mathrm{ra0} 05115 \mathrm{~b}$

rsc.li/rsc-advances these compact diagnostic devices widens the range of strategies for disease monitoring and management. ${ }^{14}$

Recently, lab-on-a-valve (LOV) devices have emerged as a new technology for flow control. ${ }^{15-17} \mathrm{LOV}$ devices have the advantage of being directly manipulated by a programmable flow-based platform that can adopt different coupling modes and controlled fluid movement, as compared to the lab-on-a-chip. ${ }^{18}$ Flow analyses by LOV devices seem to be suitable for the development of portable analysis systems in that this technology reduces instrument size and reagent and energy consumption, which are important benefits for field systems, while simplifying the work required for analysis. ${ }^{19}$

In this study, we applied flow analysis to switchable linker (SL)-based analysis using 3-way valve chambers (3-VCs). The 3way valve, which provides a convenient and economical means of selecting various flow rates and directions, plays a crucial role in almost all industrial processes, including the food field..$^{20-22}$ In addition, the 3-way valve can control the flow of fluid via a simple operation and can be applied to various fluids based on their materials, volume, and ranges of temperature and pressure. ${ }^{23,24}$ However, a 3-way valve that is sealable, portable, and easy to operate has not yet been developed for use as a reaction chamber for bioassays. In addition, both disposable syringes and syringe filters, which have the advantages of sterilization, low cost, and wide application, can comprise a good biosensing application kit in the field. ${ }^{25-28}$

Colorimetric detection based on gold-nanoparticle aggregation has attracted interest as a novel alternative for on-site detection due to its simplicity and the fact that it needs no 
sophisticated equipment or technology. ${ }^{29-32}$ The goldnanoparticle aggregation-based colorimetric method has a limitation in that its detection signal is weak when detecting a relatively large target such as a pathogen because the spectral aggregation shift decreases as the distance between gold nanoparticles increases. ${ }^{33}$ Therefore, in our previous research, we proposed the use of a switchable linker to induce the largescale aggregation of gold nanoparticles and to enhance the detection signal by sedimentation of the aggregates. This novel colorimetric detection method is expected to increase the capability of on-site detection.

In this study, we first developed a portable kit comprising a 3-way valve for the on-site detection of Salmonella Typhimurium. This kit is based on the use of colorimetric detection by the precipitation of gold nano-aggregates (Au NAs) and a novel switchable linker to amplify the visible signal by the aggregation of gold nanoparticles. ${ }^{34-37}$ In addition, for quick on-site detection, we introduced filtration into the device as a strategy for improving the visual detection signal.

\section{Experimental}

\section{Chemicals, reagents, and instruments}

We purchased streptavidin, phosphate-buffered saline (PBS), $1 \mathrm{mM}$ tetrachloroaurate, and bovine serum albumin (BSA) from Sigma-Aldrich (Madison, WI), and tetraborate $\mathrm{pH}$ standard solution from Wako Pure Chemicals (Osaka, Japan). We purchased anti-Salmonella polyclonal antibody (biotin) (ab69255) from Abcam (Cambridge, MA) and vials, ethanol, and trisodium citrate from local suppliers.

As the buffer in the synthesis of $13 \mathrm{~nm}$ Au NPs, we used sterilized distilled water, which we prepared with $1 \%(\mathrm{w} / \mathrm{v})$ tri-sodium citrate as a reducing agent. The buffers used for the SL-based assay for the detection of streptavidin as a model target and Salmonella Typhimurium were $0.01 \mathrm{~mol} \mathrm{~L}^{-1} \mathrm{PBS}(\mathrm{pH} 7.4)$ prepared with $0.1 \%$ and $0.5 \%(\mathrm{w} / \mathrm{v}) \mathrm{BSA}$, respectively, for blocking, and $0.01 \mathrm{~mol} \mathrm{~L}^{-1}$ tetraborate $\mathrm{pH}$ standard solution $(\mathrm{pH}$ 7.4) for diluting the streptavidin. The bacterial strain used in the assay was Salmonella Typhimurium (ATCC 19585) which was purchased from ATCC.

We performed the SL-based immunoassay in 3-VCs purchased from a local supplier, and filtered the Au NAs using a $50 \mathrm{~mL}$ syringe (local supplier) and syringe filters (Hyundai Micro, South Korea). To determine the assay absorbance, we used a UV-1700 spectrophotometer (Shimadzu, Japan). The size (number) of the Au NAs was determined using a Zetasizer (Nano ZS, Malvern Instruments Ltd., Worcestershire, UK).

\section{Preparation of colloidal streptavidin-coated Au NPs}

We prepared colloidal Au NPs with an average size of $13 \mathrm{~nm}$ by reducing tetrachloroaurate with tri-sodium citrate, which is a well-established citrate reduction method. ${ }^{38}$ We verified the particle size by transmission electron microscopy (TEM, JEM1010, JEOL, Japan) and UV-Vis spectroscopy (UV-1700 PC, Shimadzu). The synthesized Au NPs had an ultraviolet-visible spectrum peak $\left(\lambda_{\max }\right)$ at $520 \pm 1.0 \mathrm{~nm}$. Based on the absorbance value ( 0.4 in a 1/10 dilution sample), the concentration of the Au NPs was estimated to be $\sim 7 \times 10^{12}$ particles per $\mathrm{mL}$. The prepared Au NPs were coated with streptavidin for surface functionalization using an electrostatic adsorption procedure. ${ }^{39}$ To functionalize the prepared Au NPs, we coated $40 \mu \mathrm{g}$ of streptavidin dissolved in a tetraborate solution ( $\mathrm{pH} 7.4$ ) onto $600 \mu \mathrm{L}$ of the citrate-stabilized $\mathrm{Au}$ NPs with an absorbance of 0.4 (1/10 dilution sample) at $520 \pm 1.0 \mathrm{~nm}$. To remove unbound streptavidin, centrifugation was conducted at $10000 \mathrm{~g}$ for $30 \mathrm{~min}$. Then, the supernatant containing the unbound streptavidin was removed and washed off and then mixed with $0.5 \%(\mathrm{w} / \mathrm{v})$ BSA dissolved in PBS as a blocking buffer. The Au NPs coated with streptavidin molecules (st-Au NPs) exhibited a peak at $531.5 \pm$ $0.5 \mathrm{~nm}$ at a concentration with a peak absorbance value of 0.4 (1/ 10 dilution sample) in their UV-Vis spectra.

\section{Fabrication of the portable kit}

The major objective in developing the prototype kit was to produce a low-cost portable reaction chamber with a 3-way valve to provide an easy-to-use device for primary diagnosis. As shown in Fig. 1, the portable kit consists of a 3-way valve that acts as a reaction chamber, a $50 \mathrm{~mL}$ syringe that is used for sample injection and as a filter press, and a syringe filter that filters out $\mathrm{Au}$ NAs to obtain a rapid detection signal. To store $200 \mu \mathrm{L}$ of streptavidin-coated Au NPs (st-Au NPs) and handle the total volume of $400 \mu \mathrm{L}$ in the reaction, we determined the required pipe length and diameter of the $3-\mathrm{VC}$ to be $16 \mathrm{~mm}$ and $4 \mathrm{~mm}$, respectively. To minimize interaction of the device material with proteins, we selected polyvinylidene fluoride (PVDF, hydrophilic) as the 3 -VC material.

\section{Optimization of the syringe filter}

In the SL-based assay, the visible detection signal is amplified by the loss of colour following the precipitation of the large scale-aggregates of Au NPs induced by the SL. In this study,

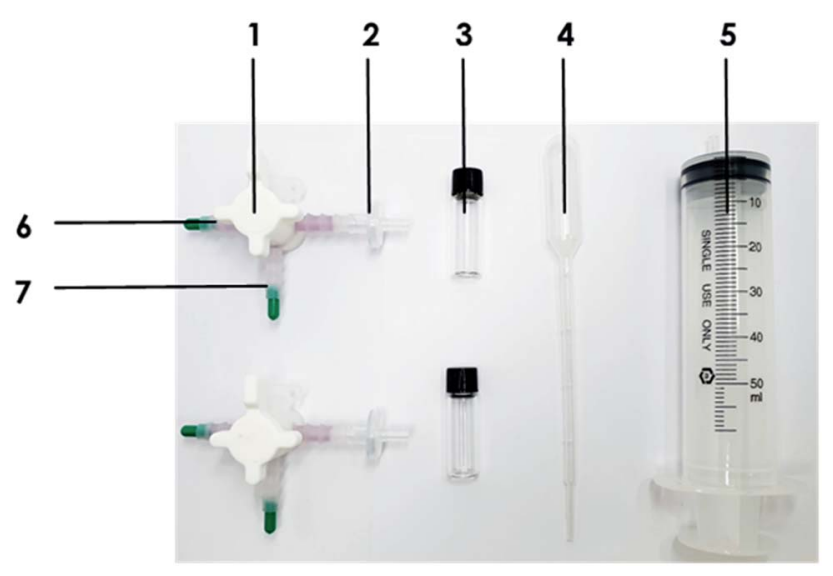

Fig. 1 Photograph of the portable detection kit, which consists of the following materials and reagents: (1) 3-way valve that serves as a storage and reaction chamber, (2) a syringe filter $(0.45 \mu \mathrm{m}, \mathrm{PVDF}$, hydrophilic), (3) a signal-indication vial, (4) a disposable pipette used to inject the test sample, (5) a $50 \mathrm{~mL}$ syringe for applying pressure to the filter, (6) the reaction chamber containing the linker (b-Ab) solution, and (7) the storage chamber containing the st-Au NP solution. 
since a filter was introduced to quickly obtain the detection result, experiments were conducted to compare the performances of various filters and determine which was most effective. As shown in Table 1, filters comprising various materials (mixed cellulose ester, polyethersulfone, cellulose acetate, polyvinylidene fluoride, polyamide, and polytetrafluoethylene) and sizes $(0.2 \mu \mathrm{m}$ and $0.45 \mu \mathrm{m})$ were used in the filtration of Au NAs in PBS, milk, and $0.1 \%$ milk. The total $400 \mu \mathrm{L}$ volume of the st$\mathrm{Au}$ NP solution was filtered through each syringe filter.

\section{Detection procedure}

The SL-based assay, developed in a previous study, was performed using the portable kit. As shown in Fig. 2C and D, SLbased assays were performed in a total reaction volume of 400 $\mu \mathrm{L}$ containing $200 \mu \mathrm{L}$ of st-Au NP solution at a fixed concentration, $100 \mu \mathrm{L}$ of biotinylated polyclonal Salmonella antibody (b-Ab) as the SL, and $100 \mu \mathrm{L}$ of a test sample (PBS at pH 7.4). First, $100 \mu \mathrm{L}$ of $\mathrm{b}-\mathrm{Ab}$ was prepared in a closed reaction chamber of the 3 -VC and $200 \mu \mathrm{L}$ of st-Au NPs was prepared in the closed pipe of the 3-VC. One hundred microliter of the test sample was injected into one pipe of the 3-VC using the syringe, and then this 3-VC mixture was turned and shaken several times. After an adequate reaction time, we opened the valve to allow the mixture to react with the st-Au NPs. After a sufficient reaction time, we filtered the Au NAs through the syringe filter $(0.45 \mu \mathrm{m}$, PVDF) to obtain a visible detection signal.

Prior to performing the SL-based assays for the detection of Salmonella Typhimurium, we had to determine the range of the SL (b-Ab) concentration exhibiting visible colour change (REVC). An assay was performed in PBS at various b-Ab concentrations $\left(10-45 \mu \mathrm{g} \mathrm{mL}{ }^{-1}\right)$. Based on the REVC results, we selected the concentrations of 13 to $15 \mu \mathrm{g} \mathrm{mL}{ }^{-1}$ to be the most effective in clearly showing the colour change at the low end of REVC.

\section{Optimization of secondary reaction time for filtration}

To filter out the Au NAs, the change in the sizes of the Au NAs over time was measured using a UV-Vis spectrometer and a Zetasizer, respectively. As SLs, 10 and $13 \mu \mathrm{g} \mathrm{mL} \mathrm{m}^{-1}$ of biotinylated Salmonella polyclonal antibodies were used as control and aggregates samples, respectively. We added $200 \mu \mathrm{L}$ of st-Au NP to each test samples and tracked the sizes every 2 min using a Zetasizer.

\section{Statistical analysis}

The data represent the average of at least three independent experiments or measurements.

\section{Results and discussion}

\section{Optimization of 3-way valve chamber (3-VC)}

To apply the 3-VC to the SL-based assay, it was necessary to optimize the material, the pore and bore sizes of the tubes, the maximum volume, and the loss volume (Table 2). To minimize friction with the solution, among the 3 -VC materials considered were polyvinylidiene fluoride (PVDF), polypropylene/ polyethylene (PP/PE), and polyvinyl chloride. Ultimately, PVDF was selected due to its low loss volume with the system solution for every pore size. To select the final volume of the 3-way valve, using inner and outer diameters of $4 \mathrm{~mm}, 6 \mathrm{~mm}$, and $8 \mathrm{~mm}$ and $6 \mathrm{~mm}, 8 \mathrm{~mm}$, and $10 \mathrm{~mm}$, respectively, we considered the compatibility of the syringes and syringe filters and their application to the assay. As they were found to be applicable to the assay under all volume conditions, we selected a valve with a $4 \mathrm{~mm}$ inner diameter and $6 \mathrm{~mm}$ outer diameter that was compatible with the syringe and syringe filter. Finally, a 3-way valve consisting of PVDF material with an inner diameter of $4 \mathrm{~mm}$ and an outer diameter of $6 \mathrm{~mm}$ was finally determined to be most suitable as a portable device for field application of the SL-based assay.

\section{The SL-based immunosensing mechanism by 3-VC}

Fig. 2 shows the proposed SL-based immunosensing mechanism for the on-site detection of Salmonella Typhimurium using the developed kit. The SL-based assay can induce the aggregation of st-Au NP with b-Ab and detect the target bacteria in a real matrix. As shown in Fig. $2 \mathrm{~A}$ and $\mathrm{B}$, we designed the SL

Table 1 Comparison of materials, pore sizes, and filtering abilities of the syringe filters

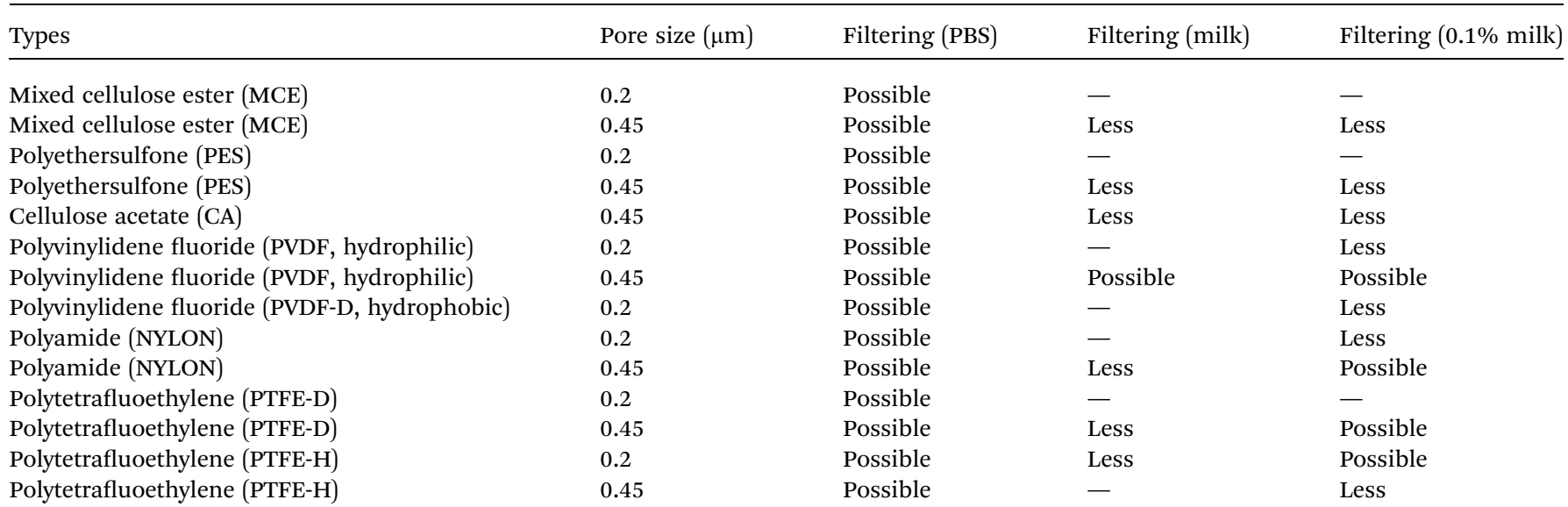


(A)

(B)

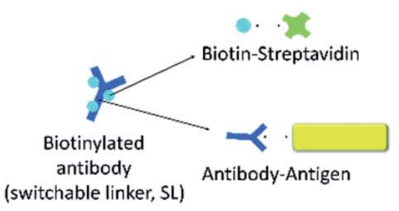

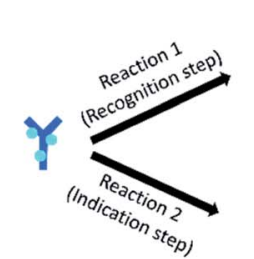

(D)

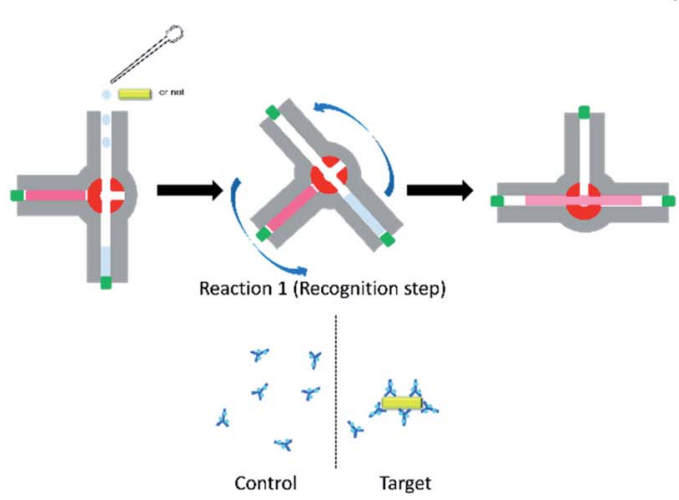

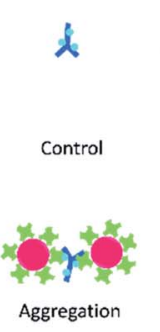
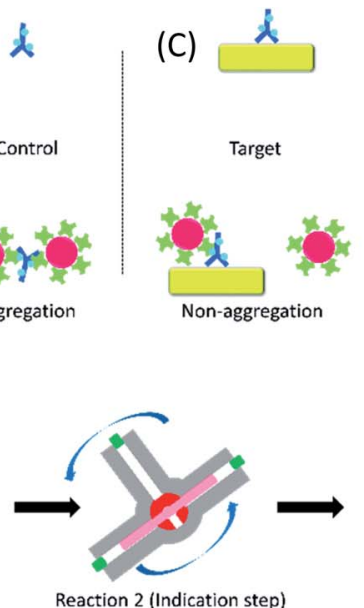

Reaction 2 (Indication step)

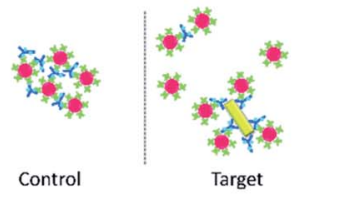

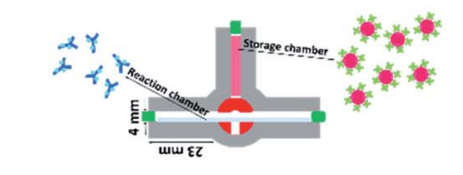

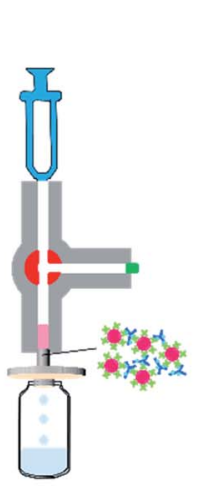

Control

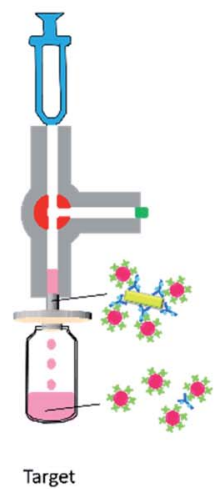

Target

Y Salmonella antibody $\odot$ Biotin 4 Streptavidin Salmonella Typhimurium

Gold nanoparticle (Au NP)

Fig. 2 Schematic of detection principles and procedure. (A) Biotinylated Salmonella antibody (b-Ab) can bridge to st-Au NP (by the biotinstreptavidin interaction) and target Salmonella (via the antibody-antigen interaction). (B) The SL-based assay is performed in two sequential steps: the first reaction serves as a target recognition step, in which SL reacts with the target bacteria. The second reaction serves as a signal indication step, in which SL reacts with st-Au NPs. (C) The configuration of the 3-VC comprising the reaction chamber with SLs and the storage chamber with st-Au NPs. (D) The procedure of the SL-based assay in the 3-VC.

(biotinylated antibody) to have two reactions: target recognition (the antibody-antigen immune response) and signal recognition (biotin-streptavidin binding response). Generally, the SLbased assay is performed in two sequential steps. First, the target and the SL undergo a sufficient reaction. Thereafter, depending on the presence or absence of the target, the quantitatively readjusted SLs react with st-Au NPs. When the SLs are bound to their targets, their ability to cross-link with st-Au NP is limited. We refer to this state as the linker being switched-off. Therefore, since the SL is switched off by the target, the degree of aggregation of the st-Au NPs is changed, so the SLbased assay can exhibit a visible colour-fading signal depending on the presence or absence of the target.

In this study, a 3-VC was introduced in the proposed kit. As shown in Fig. 2C, the 3-VC consists of a storage chamber and a reaction chamber. The storage chamber contains $200 \mu \mathrm{L}$ of st$\mathrm{Au}$ NPs solution and the reaction chamber contains $100 \mu \mathrm{L}$ of biotinylated Salmonella antibody (b-Ab) solution. The amount of SL required to induce aggregation of st-Au NPs depends on the 'concentration' of the target in the system. There is also a range of SL concentrations for a given concentration of the target and st-Au NPs that leads to large-scale aggregation and a visible colour change by precipitation of the aggregates, which is known as the REVC. Therefore, the SL can be distinguished as having two regions, i.e., the REVC and the region with no aggregation of st-Au NPs and no colour change (more specifically, the SL concentration range can actually be divided into three parts, which are not explained here). The SL-based assay can identify the target organism based on the REVC shift in response to the 'switching-off' of the SL by the target.

\section{System stability of $\mathrm{pH}$ and salt conditions}

Various and complex components in food are likely to interfere with the detection system. In particular, various $\mathrm{pH}$ and high

Table 2 Comparison of the materials, pore size, total volume, loss volume, and REVC signal of a 3-VC for SL-based assay

\begin{tabular}{llllll}
\hline Materials & Diameter (pore, $\mathrm{mm})$ & Diameter $($ bore, $\mathrm{mm})$ & Max volume $(\mu \mathrm{L})$ & Loss volume $(\mu \mathrm{L})$ & REVC signal \\
\hline PP/PE & 6 & 4 & 500 & $<120$ & Work \\
& 8 & 6 & 1200 & $<220$ & Work \\
PVDF & 10 & 8 & 4000 & $<380$ & Work \\
& 6 & 4 & 500 & $<100$ & Work \\
& 10 & 6 & 1200 & $<190$ & Work \\
& 8 & 8 & 4000 & $<290$ & Work
\end{tabular}


salt concentrations in foods are one of the main causes of biosensor inhibition. In particular, the main factor of Au NP aggregation in this detection system is biotin-streptavidin binding force. In areas where this system will be used, there is no way to remove salt and adjust the $\mathrm{pH}$ of the system, so these harsh conditions have to be checked. Streptavidin is a protein as known for its high thermal and mechanical stability. Therefore, stability testing of this system was conducted with emphasis on salt and $\mathrm{pH}$ resistance. As shown in Fig. 3, the system confirmed the REVC shift of the control under all $\mathrm{pH}$ and salt conditions. As a result, the system was stable under all conditions used in the experiment.

The stabilization study of this system was conducted over a wide range of linker concentrations, which can be divided into three regions associated by the size of the aggregates. The size of aggregates was determined by a quantitative correlation between the Au NP and the linker. In the two regions of the linker concentrations of 10 to $15 \mu \mathrm{g} \mathrm{mL}^{-1}$ and $40 \mu \mathrm{g} \mathrm{mL} \mathrm{m}^{-1}$ as shown in Fig. 3, the size of the aggregates of the Au NPs was small so that precipitation did not completely occur. In the case of the linker concentration of $20 \mu \mathrm{g} \mathrm{mL} \mathrm{m}^{-1}$, the size of Au NAs was large enough to precipitate completely. This was explained in previous system studies.

There are various components including sugars, proteins, and lipids in real food matrices, and the operation of the system may be inhibited by those things. However, since this system sets a control sample without a target, even if the real food matrix affects the system operation, the REVC in control sample could be reset. Therefore, it can be effectively detected of pathogens if the REVC could be shifted by only a target. In addition, in previous studies, we reported that the SL-based assay could work in serum as well as in various food matrices such as tomato and milk. ${ }^{34-37}$ Therefore, we dealed with the minimum environmental factors (salt and $\mathrm{pH}$ ) in this study.

\section{Determination of reaction time (primary reaction time) for targets to crosslink with switchable linkers}

The SL used in this study was designed based on an antibody with biotins, and unlike the ELISA, a specific SL concentration

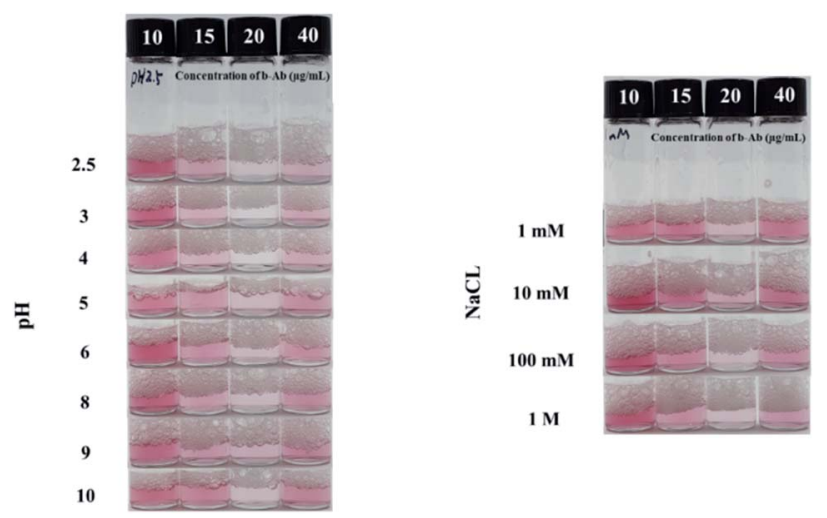

Fig. 3 REVC shift of the control using 3-way valve chamber and syringe filter at various system conditions: (A) $\mathrm{pH} 2.5,3,4,5,6,8,9,10$, (B) 1, 10, $100 \mathrm{mM}$, and $1 \mathrm{M}$ of $\mathrm{NaCL}$. is used in the SL-based assay without any washing step. Therefore, it is necessary to confirm the activity of the SL reacting with the target. In doing so, we tried to optimize the first reaction time of this assay, i.e., the reaction between the SL and the target in the 3-VC. As shown in Fig. 4, $10 \mu \mathrm{g} \mathrm{mL} \mathrm{m}^{-1}$ of target Salmonella Typhimurium reacted with $10 \mu \mathrm{g} \mathrm{mL}^{-1}$ to 40 $\mu \mathrm{g} \mathrm{mL} \mathrm{m}^{-1}$ of b-Ab as SLs for $0 \mathrm{~min}, 15 \mathrm{~min}, 30 \mathrm{~min}$, and $60 \mathrm{~min}$, and then the st-Au NPs were treated to observe the change in the REVC. After $30 \mathrm{~min}$, an REVC shift was observed at a linker concentration of $13 \mu \mathrm{g} \mathrm{mL}{ }^{-1}$.

\section{Determination of reaction time (secondary reaction time) for producing the REVC signal using filter}

Switchable linkers can play the roles of both crosslinking the $\mathrm{Au}$ NPs and binding the target bacteria, both of which are mutually limited.

In other words, in the sequential steps of the SL-based assay, when the SL is combined with the target bacteria, it is switched off and plays no role in inducing aggregation of gold nanoparticles. The SL that is not bound to the target induces largescale aggregation of gold nanoparticles, which can eventually produce a visual signal whose colour fades as it slowly precipitates out over time (up to $2 \mathrm{~h}$ without shaking). The second step of this assay, which determines the REVC, consists of an aggregation reaction of the combination of streptavidin and biotin and a precipitation reaction based on the size of the $\mathrm{Au}$ NAs, the latter being the rate-determining step. Therefore, in this study, a filter was applied to the SL-based assay to filter out $\mathrm{Au}$ NAs of a certain size to minimize the time required for precipitation and thereby obtain visual signals more quickly.

To filter out Au NAs, it was necessary to track the size of the aggregates. As shown in Fig. 5A and B, Au NA was filtered out for

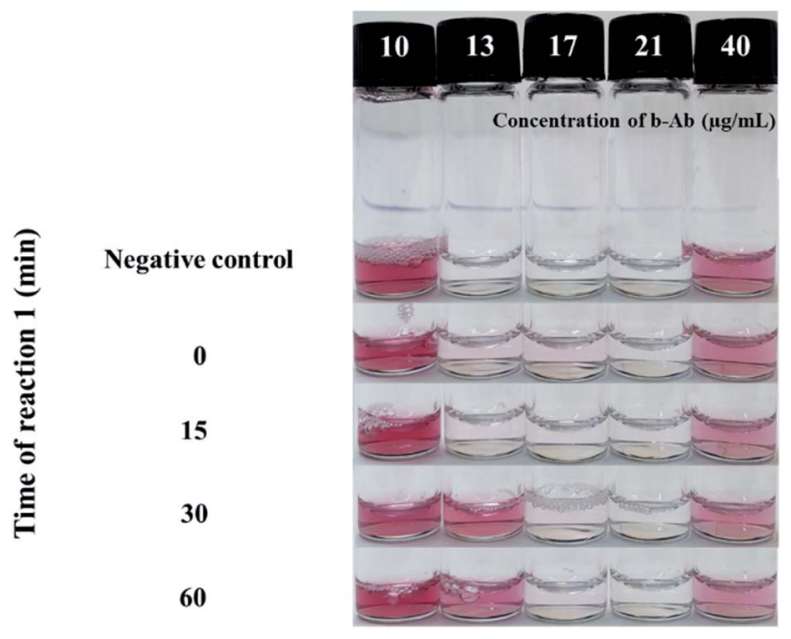

Fig. 4 Photograph showing the effect of reaction time 1 with the negative control and Salmonella Typhimurium. Shift in REVC with increasing reaction times between $\mathrm{b}-\mathrm{Ab}$ and the presence of $10^{5} \mathrm{CFU}$ $\mathrm{mL}^{-1}$ of Salmonella Typhimurium for $120 \mathrm{~min}$ after adding $100 \mu \mathrm{L}$ of st-Au NPs (absorption: 0.4 a $530 \pm 0.5 \mathrm{~nm}$ ) in a $400-\mu \mathrm{L}$ total test system volume using the $3-\mathrm{VC}$ to determine the optimal time of $1^{\text {st }}$ reaction. After $30 \mathrm{~min}$, the REVC shifts at a linker concentration of 13 $\mu \mathrm{g} \mathrm{mL}{ }^{-1}$. 
$15 \mathrm{~min}$ at an SL concentration of $10 \mu \mathrm{g} \mathrm{mL} \mathrm{m}^{-1}$ in cases in which no large aggregation was formed and $13 \mu \mathrm{g} \mathrm{mL} \mathrm{m}^{-1}$ in cases in which large aggregations were formed. The time to visually recognize that the colour had faded was about $4 \mathrm{~min}$, and after 6 min the solution became clear enough to notice the difference in colour completely. As a result of tracking the size of Au NA for $15 \mathrm{~min}$ in $2 \mathrm{~min}$ increments by a Zetasizer, it took $7 \mathrm{~min}$ for the size of $\mathrm{Au}$ NA to grow as large as the size of the filter pore $(0.45$ $\mu \mathrm{m}$, the red dash line in Fig. 5C). Taken together, we finally determined that the enough time required for filtration was 7 min. Switchable linkers can crosslink Au NPs to form Au NAs. In the case of the previous SL-based assay, the colour change time can be determined by the sum of the time for forming the aggregate and the time for precipitation, in which the latter takes longer than the former. Therefore, in this study, as a result of introducing the filtration process with the aim of reducing the reaction time of the precipitation and aggregates formation time, the total detection time was greatly reduced by reducing the second step from $2 \mathrm{~h}$ to $7 \mathrm{~min}$. This result is expected to introduce a simple syringe filter as part of the kit so that the SLbased assay can detect food-borne pathogens rapidly in the field.

\section{Evaluation experiments}

To evaluate the performance of the developed device, we used it to detect Salmonella Typhimurium on the basis of the optimized parameters at various target concentrations. Salmonella

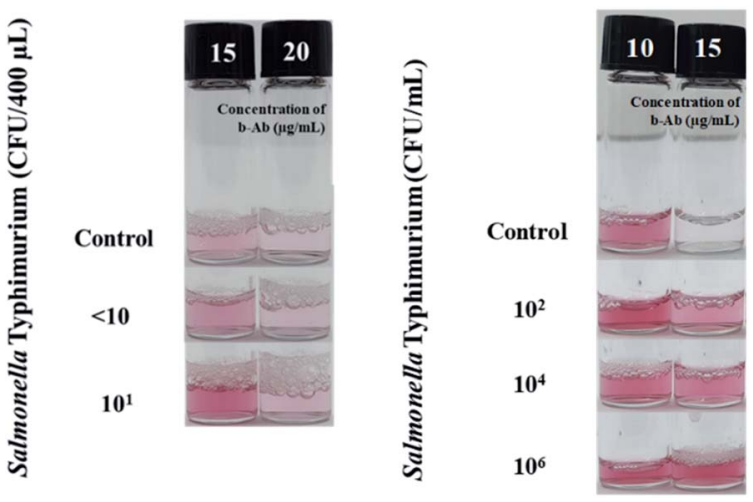

Fig. 6 Evaluation of the SL-based immunoassay to the detection of S. Typhimurium using the developed device. The REVC shift at low end after performing the SL-based assay for on-site detection of $S$. Typhimurium $\left(0,<10,10^{1}, 10^{2}, 10^{4}, 10^{6} \mathrm{CFU} \mathrm{mL}^{-1}\right)$.

Typhimurium, which confirmed the selectivity of SL-based assay in the previous study, was used as a representative strain. ${ }^{35}$ As shown in Fig. 6, the REVC shift occurred at a linker concentration of $15 \mu \mathrm{g} \mathrm{mL}{ }^{-1}$, as compared to the control in the tested Salmonella Typhimurium samples $\left(10^{2}, 10^{4}\right.$, and $10^{6} \mathrm{CFU} /$ $400 \mu \mathrm{L}$ ). The REVC shift occurred at a linker concentration of 15 $\mu \mathrm{g} \mathrm{mL}{ }^{-1}$, as compared to the control in all the tested Salmonella Typhimurium samples. In addition, to identify an LOD, Salmonella Typhimurium with low level of the concentrations $\left(0,<10,10^{1} \mathrm{CFU} / 400 \mu \mathrm{L}\right)$ were tested. The assay was evaluated by
(A)

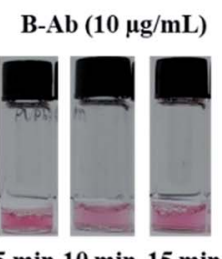

$5 \mathrm{~min} 10 \mathrm{~min} 15 \mathrm{~min}$
(B)

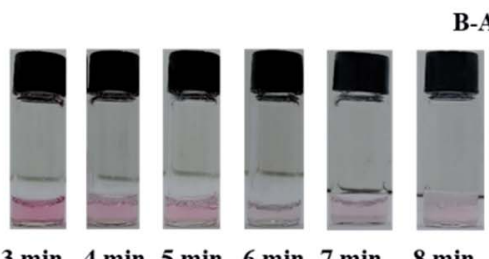

B-Ab $(13 \mu \mathrm{g} / \mathrm{mL})$
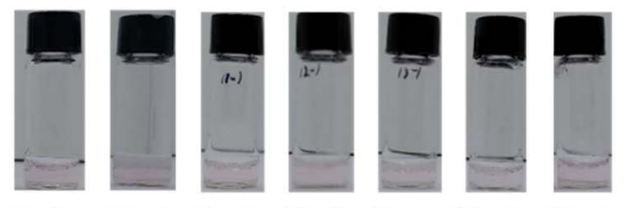

(C)

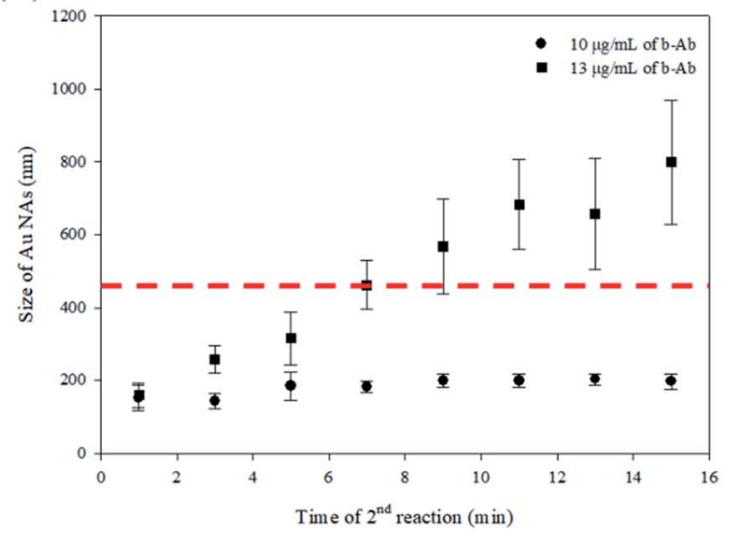

Fig. 5 Finding the optimal time of $2^{\text {nd }}$ reaction to filter the aggregates of st-Au NPs. The appearances of $2^{\text {nd }}$ reaction for 15 min at (A) 5 min increments in $10 \mu \mathrm{g} \mathrm{mL}^{-1}$ of b-Ab (no large-scale aggregation) and (B) 1 min increments in $13 \mu \mathrm{g} \mathrm{mL}^{-1}$ of b-Ab (large-scale aggregation). The tracking results of size change of Au NAs over 15 min by (C) a Zetasizer. 
the naked eye after filtering of Au NAs. As a result, the clear colour change (REVC) in $15 \mu \mathrm{g} \mathrm{mL}^{-1}$ of b-Ab was seen down to $10^{1} \mathrm{CFU} / 400 \mu \mathrm{L}$ of Salmonella Typhimurium. Therefore, the LOD of the BL-based assay using 3 -VC was determined to be $10^{1}$ $\mathrm{CFU} / 400 \mu \mathrm{L}$.

Since the conventional SL-based assay is based on the precipitation of Au NAs, up to two hours is required to confirm the results. However, in the proposed device, a filter was introduced so that when the Au NAs grow to a certain size that can be filtered, the whole detection time can be reduced by shortening the time required for settling. By applying the filter, the detection result can be quickly confirmed, but we found there to be a volume difference in the filtered system depending on the pressure applied to the filter. In addition, depending on the aggregation mechanism between the target and the gold nanoparticles for each linker concentration, there was a difference in the colour concentration because of the size of the aggregates and the number of gold nanoparticles that did not participate in the reaction. Therefore, when using the proposed apparatus, when selecting a specific linker density, it is necessary to select a section in which the difference in colour density is apparent and the REVC shift is reliably evident. By choosing linker concentrations of $10 \mu \mathrm{g} \mathrm{mL}^{-1}$ and $15 \mu \mathrm{g} \mathrm{mL}^{-1}$, Salmonella Typhimurium could be detected down to $10^{1} \mathrm{CFU} / 400 \mu \mathrm{L}$.

This study has suggested two factors to make the SL-based assay more suitable for on-site application. First, a prototype kit that can be easily applied to this assay beyond the laboratory level was proposed. Since the existing method uses reagents, such as buffers, and instruments, such as pipettes, it has application limitations in the field. Second, in order to reduce the detection time, we applied a filter to control the time required for the $\mathrm{Au}$ NAs to precipitate. In the existing assay, a shaking method was used to control the time required for precipitation, which required a device such as a vortex, or limiting its use in the field. Therefore, introduction of a 3-way valve-based kit and filter is considered to have upgraded the steps to make the SL-based assay practical.

\section{Conclusions, future directions}

Although reliable detection methods for pathogens have been developed for use in the medical, food, and environmental fields, there are many problems to overcome before they can be used in mobile field applications, including the need for miniaturization, rapid diagnosis, and low cost. In this study, we developed a prototype device for making primary diagnoses using the SL-based assay with a 3-VC and a syringe filter for the detection of Salmonella Typhimurium. The use of this assay will be very advantageous in situations where the target bacteria must be identified quickly in the field using a simple experimental procedure and a miniaturized device. It is necessary to solve problems, such as automation of each step's reaction for increasing user convenience using a mechanical and electrical instrument, massification of a wide linker concentration for quantitative detection, and stability of the system through the development of new linker such as an aptamer, in order for this strategy to be used at industrial sites. When these problems are associated with a device and system, they can be solved, and it is expected that targets, such as biological hazards in real food samples can be detected very quickly and easily.

\section{Conflicts of interest}

There are no conflicts of interest to declare.

\section{Acknowledgements}

This research was supported by the Basic Science Research Program through the National Research Foundation of Korea (NRF), which is funded by the Ministry of Education (NRF2013R1A1A2012823 and NRF-2020R1I1A1A0106444511).

\section{Notes and references}

1 K. K. Alharbi and Y. A. Al-sheikh, Saudi J. Biol. Sci., 2014, 21, 109-117.

2 Y.-F. Chen, L. Jiang, M. Mancuso, A. Jain, V. Oncescu and D. Erickson, Nanoscale, 2012, 4, 4839-4857.

3 S. Nayak, N. R. Blumenfeld, T. Laksanasopin and S. K. Sia, Anal. Chem., 2016, 89, 102-123.

4 P. Yager, G. J. Domingo and J. Gerdes, Annu. Rev. Biomed. Eng., 2008, 10, 107-144.

5 M. U. Ahmed, M. M. Hossain, M. Safavieh, Y. L. Wong, I. A. Rahman, M. Zourob and E. Tamiya, Crit. Rev. Biotechnol., 2016, 36, 495-505.

6 R. Dragone, G. Grasso, M. Muccini and S. Toffanin, Frontiers in Public Health, 2017, 5, 80.

7 S. K. Vashist, P. B. Luppa, L. Y. Yeo, A. Ozcan and J. H. Luong, Trends Biotechnol., 2015, 33, 692-705.

8 P. Mandal, A. Biswas, K. Choi and U. Pal, Am. J. Food Technol., 2011, 6, 87-102.

9 S.-H. Seo, Y.-R. Lee, J. H. Jeon, Y.-R. Hwang, P.-G. Park, D.-R. Ahn, K.-C. Han, G.-E. Rhie and K.-J. Hong, Biosens. Bioelectron., 2015, 64, 69-73.

10 L. Xiang, H. Wu, Z. Cui and J. Tang, Anal. Lett., 2019, 52, 1966-1975.

11 H. Chen, K. Liu, Z. Li and P. Wang, Clin. Chim. Acta, 2019, 493, 138-147.

12 C. D. Chin, T. Laksanasopin, Y. K. Cheung, D. Steinmiller, V. Linder, H. Parsa, J. Wang, H. Moore, R. Rouse and G. Umviligihozo, Nat. Med., 2011, 17, 1015.

13 P. Yager, T. Edwards, E. Fu, K. Helton, K. Nelson, M. R. Tam and B. H. Weigl, Nature, 2006, 442, 412.

14 G. M. Whitesides, Nature, 2006, 442, 368.

15 M. L. de Castro, J. Ruiz-Jiménez and J. Pérez-Serradilla, TrAC, Trends Anal. Chem., 2008, 27, 118-126.

16 J. Ruzicka, Analyst, 2000, 125, 1053-1060.

17 J. Wang and E. H. Hansen, TrAC, Trends Anal. Chem., 2003, 22, 225-231.

18 M. Miró and E. H. Hansen, Anal. Chim. Acta, 2012, 750, 3-15. 19 E. Mattio, F. Robert-Peillard, L. Vassalo, C. Branger, A. Margaillan, C. Brach-Papa, J. Knoery, J.-L. Boudenne and B. Coulomb, Talanta, 2018, 183, 201-208. 
20 P. Athanasatos and T. Costopoulos, Mechanism and Machine Theory, 2012, 50, 64-89.

21 M. Giousouf and G. Kovacs, Smart Mater. Struct., 2013, 22, 104010.

22 M. L. S. Silva, M. B. Q. Garcia, J. L. Lima and E. Barrado, Talanta, 2007, 72, 282-288.

23 W. J. Kitsch, US Pat., 7895 850, 2011.

$24 \mathrm{~S}$. Shoji, in Microsystem technology in chemistry and life science, Springer, 1998, pp. 163-188.

25 J. Fang, Q. Wang and N. Hu, in Micro/Nano Cell and Molecular Sensors, Springer, 2016, pp. 97-123.

26 T. C. Hinkley, S. Singh, S. Garing, A.-L. M. Le, K. P. N. Ny, J. E. Peters, J. N. Talbert and R. Sam, Bioengineering Bacteriophage for the Sensitive \& Rapid Detection of Bacteria in Water, 2018, p. 147.

27 H. E. Indyk, E. A. Evans, M. C. B. Caselunghe, B. S. Persson, P. M. Finglas, D. C. Woollard and E. L. Filonzi, J. AOAC Int., 2000, 83, 1141-1148.

28 L. Y. Yeo, H. C. Chang, P. P. Chan and J. R. Friend, Small, 2011, 7, 12-48.

29 G.-H. Chen, W.-Y. Chen, Y.-C. Yen, C.-W. Wang, H.-T. Chang and C.-F. Chen, Anal. Chem., 2014, 86, 6843-6849.
30 Z. Guo, J. Duan, F. Yang, M. Li, T. Hao, S. Wang and D. Wei, Talanta, 2012, 93, 49-54.

31 Y. Luo, J. Xu, Y. Li, H. Gao, J. Guo, F. Shen and C. Sun, Food Control, 2015, 54, 7-15.

32 S. E. Magubane, S. Ntlhoro, M. Sabela, S. Kanchi, M. Mlambo, S. C. Onwubu, P. S. Mdluli and A. M. Asiri, J. Taiwan Inst. Chem. Eng., 2019, 101, 159-166.

33 I. E. Sendroiu, S. F. Mertens and D. J. Schiffrin, Phys. Chem. Chem. Phys., 2006, 8, 1430-1436.

34 J. Hahn, E. Kim, Y. S. You and Y. J. Choi, Analyst, 2019, 144, 4439-4446.

35 J. Hahn, E. Kim, Y. S. You, S. Gunasekaran, S. Lim and Y. J. Choi, J. Food Sci., 2017, 82, 2321-2328.

36 S. Lim, O. K. Koo, Y. S. You, Y. E. Lee, M.-S. Kim, P.-S. Chang, D. H. Kang, J.-H. Yu, Y. J. Choi and S. Gunasekaran, Sci. Rep., 2012, 2, 456.

37 Y. You, S. Lim, J. Hahn, Y. J. Choi and S. Gunasekaran, Biosens. Bioelectron., 2018, 100, 389-395.

38 G. Frens, Nat. Phys. Sci., 1973, 241, 20.

39 K. C. Grabar, R. G. Freeman, M. B. Hommer and M. J. Natan, Anal. Chem., 1995, 67, 735-743. 